\title{
Quadrupole interactions in cubic rare earth intermetallics
}

\author{
P. Morin, J. Rouchy, D. Schmitt and E. du Tremolet de Lacheisserie \\ Laboratoire Louis-Néel, C.N.R.S., 166X, 38042 Grenoble Cedex, France
}

\begin{abstract}
Résumé. - Cette vieille science qu'est la magnétoélasticité trouve actuellement un plein renouveau à propos de l'étude des composés cubiques de terres rares. Il est en effet nécessaire de développer un formalisme quantique, les hypothèses classiques tombant du fait de l'anisotropie de l'aimantation. La variation du coefficient magnétoélastique est suivie à travers les séries RZn et RSb. Une meilleure compréhension des mécanismes d'aimantation est permise en considérant la magnétostriction et l'échange quadrupolaire.
\end{abstract}

\begin{abstract}
Magnetoelasticity in presence of crystalline electric field has recently received a great development. Indeed, cubic rare earth intermetallics exhibit large lattice distortions appearing through either a magnetostrictive process or a cooperative Jahn-Teller mechanism. Due to the anisotropic reduction of the moment, the classical theory falls and one has to develop a quantum treatment. The variation of the magnetoelastic coefficient is analyzed throughout the $\mathrm{RSb}$ and $\mathrm{RZn}$ series. Taking into account magnetostriction and quadrupolar exchange allows then a good description of the magnetization processes.
\end{abstract}

1. Introduction. - Magnetoelastic effects and in a more general way magnetic quadrupolar interactions have been extensively studied over the last few years in rare earth intermetallics. Two reasons may be proposed for this revival of the old science of magnetoelasticity : firstly the observed lattice strains are strong enough for suggesting possible technological applications [1]. Secondly, the classical treatment based on the isotropic moment modulus fails in presence of a strong crystal electric field (CEF) [2]. Hence a quantum description for magnetoelasticity must be developped (e.g. cubic compounds with $\mathrm{NaCl}$ [3] and $\mathrm{CsCl}$ [4] structures) as for interpreting the anisotropies of the moment and the energy observed on the magnetization processes in cubic rare earth intermetallics [5].

We first want to describe the Hamiltonian for understanding both magnetic dipole and quadrupole properties in cubic symmetry. Special attention will be paid to the parastriction. We then present some characteristic examples focusing on two series having simple structure, namely RSb and RZn. The separation of the magnetoelastic contribution from the quadrupolar exchange is discussed before analyzing the variation of the magnetoelastic coefficient throughout the series. The effects of these two second-order terms on the magnetization processes will be then illustrated.

2. Mathematical treatments. - It is well known from Trammell [6] and Callen and Callen [2] that the classical treatment of magnetism and magnetoelasticity is based on the isotropy of the moment modulus. The $4 \mathrm{f}$ wave functions having the pure
$\left|J_{z}\right\rangle$ character, an analytical expression is then possible for the free energy. In the presence of a crystal field, the $\left|J_{z}\right\rangle$ wave functions are mixed and an anisotropic reduction of the moment appears besides the anisotropy of the energy. The higher the symmetry, the stronger the mixing. In cubic compounds, there exist various couplings favoring wave function purification : obviously the magnetoelastic one, which lowers the symmetry, but also Heisenberg exchange and Zeeman couplings. The following criterion may be then proposed : when the ratio of Heisenberg interactions to the total CEF level spacing is large, the classical hypothesis remains valid, in other cases one has to start from the basic Hamiltonian.

2.1 BASIC HaMiLTONIAN. - It may be written using the operator equivalent theorem [7] and the molecular field (MF) approximation as :

$$
\begin{gathered}
\mathcal{H}=\mathscr{H}_{\mathrm{CEF}}-g_{\mathrm{J}} \mu_{\mathrm{B}}\left(\mathbf{H}_{\mathrm{ex}}+\mathbf{H}\right) \mathbf{J}+\mathcal{H}_{\mathrm{Q}}+\mathcal{H}_{\mathrm{me}}+E_{\mathrm{e}} \\
\mathscr{H}_{\mathrm{CEF}}=A_{4}\left\langle r^{4}\right\rangle \beta_{\mathrm{J}} O_{4}+A_{6}\left\langle r^{6}\right\rangle \gamma_{\mathrm{J}} O_{6}= \\
=\frac{W x}{F_{4}} O_{4}+\frac{W(1-|x|)}{F_{6}} O_{6}
\end{gathered}
$$

is the usual cubic CEF Hamiltonian in the fourfold axes system [8]. In the MF model

$$
\mathbf{H}_{\mathrm{ex}}=\frac{3 k_{\mathrm{B}} \theta^{*}\langle\mathbf{J}\rangle}{g_{\mathrm{J}} \mu_{\mathrm{B}} J(J+1)}
$$

and $\theta^{*}$ measures the strength of the Heisenberg 
interactions ; $H$ is the applied magnetic field corrected for the demagnetizing effects

$$
\begin{gathered}
\mathscr{H}_{\mathrm{Q}}=-K_{1}\left(\left\langle O_{2}^{0}\right\rangle O_{2}^{0}+3\left\langle O_{2}^{2}\right\rangle O_{2}^{2}\right)- \\
-4 K_{2}\left(\left\langle P_{x y}\right\rangle P_{x y}+\left\langle P_{y z}\right\rangle P_{y z}+\left\langle P_{z x}\right\rangle P_{z x}\right)
\end{gathered}
$$

is the quadrupolar exchange Hamiltonian, $\mathrm{O}_{2}^{0}, \mathrm{O}_{2}^{2}$ and $P_{i j}$ being the second order Stevens operators

$$
\begin{aligned}
\mathscr{H}_{\mathrm{me}}=-B_{1}\left(\varepsilon_{3} O_{2}^{0}+\sqrt{3} \varepsilon_{2} O_{2}^{2}\right)- \\
-B_{2}\left(\varepsilon_{x y} P_{x y}+\varepsilon_{y z} P_{y z}+\varepsilon_{z x} P_{z x}\right)
\end{aligned}
$$

is the one-ion magnetoelastic Hamiltonian, linear in strain and limited to the second order as is usually accepted [9-11]. The $\varepsilon$ 's are the symmetrised external strain modes. Taking into account the elastic energy $E_{\mathrm{e}}$ and minimising the free energy $F=-k_{\mathrm{B}} T \ln Z$ relative to the $\varepsilon$ 's leads to :

$$
\begin{aligned}
\varepsilon_{3}= & \frac{1}{\sqrt{6}}\left(2 \varepsilon_{z z}-\varepsilon_{x x}-\varepsilon_{y y}\right)=\sqrt{\frac{2}{3}}\left(\frac{c}{a}-1\right)= \\
= & \frac{B_{1}}{\left(C_{11}-C_{12}\right)_{0}}\left\langle O_{2}^{0}\right\rangle \\
\varepsilon_{2}= & \frac{1}{\sqrt{2}}\left(\varepsilon_{x x}-\varepsilon_{y y}\right)=\frac{B_{1} \sqrt{3}}{\left(C_{13}-C_{12}\right)_{0}}\left\langle O_{2}^{2}\right\rangle \\
\varepsilon_{i j}= & \frac{B_{2}}{4 C_{440}}\left\langle P_{i j}\right\rangle
\end{aligned}
$$

where the $C_{0}^{\Gamma}$ are the background elastic constants without magnetic interactions. After substitution of the equilibrium $\varepsilon$ values in (1) and using a MF treatment $\mathscr{H}_{\mathrm{Q}}$ and $\mathscr{H}_{\mathrm{me}}$ appear to be isomorphous and thus indistinguishable :

$$
\begin{gathered}
\mathscr{H}_{\mathrm{Q}}+\mathscr{H}_{\mathrm{me}}=-G_{1}\left(\left\langle O_{2}^{0}\right\rangle O_{2}^{0}+3\left\langle O_{2}^{2}\right\rangle O_{2}^{2}\right)- \\
-G_{2}\left(\left\langle P_{x y}\right\rangle P_{x y}+\left\langle P_{y z}\right\rangle P_{y z}+\left\langle P_{z x}\right\rangle P_{z x}\right)
\end{gathered}
$$

with

$$
G_{1}=K_{1}+\frac{B_{1}^{2}}{\left(C_{11}-C_{12}\right)_{0}}
$$

and

$$
G_{2}=4 K_{2}+\frac{B_{2}^{2}}{4 C_{44_{0}}} .
$$

2.2 MaGNETIC AND STRAIN SUSCEPTIBILITIES, ELASTIC CONSTANTS. - First order perturbation theory leads to their expressions. The CEF paramagnetic susceptibility is defined as:

$$
\begin{aligned}
\chi_{\mathrm{CEF}}^{M}=-\frac{\partial^{2} F}{\partial H^{2}} & =-\left\langle\frac{\partial^{2} E}{\partial H^{2}}\right\rangle+ \\
+ & \frac{1}{k T}\left\langle\left(\frac{\partial E}{\partial H}\right)^{2}\right\rangle-\frac{1}{k T}\left\langle\frac{\partial E}{\partial H}\right\rangle^{2} .
\end{aligned}
$$

In the presence of Heisenberg exchange, the usual susceptibility

$$
\chi_{(\mathrm{T})}^{M}=\frac{\chi_{\mathrm{CEF}}^{M}}{1-\left(\theta^{*} / C\right) \chi_{\mathrm{CEF}}^{M}}
$$

must diverge at the ordering temperature $T_{\mathrm{c}}$ and $\theta^{*} / C=\chi_{\mathrm{CEF}}^{M}\left(T_{\mathrm{c}}\right)^{-1}$ gives for each CEF scheme a relation between $\theta^{*}$ and $T_{\mathrm{c}}, C$ being the free ion Curie constant.

In the same way, each strain susceptibility may be written as

$$
\begin{aligned}
& \chi_{\mathrm{CEF}}^{\Gamma}=-\frac{1}{\left(B^{I}\right)^{2}} \frac{\partial^{2} F}{\partial \varepsilon^{T^{2}}}=-\frac{1}{\left(B^{I}\right)^{2}} \times \\
& \times\left[\left\langle\frac{\partial^{2} E}{\partial \varepsilon^{I 2}}\right\rangle-\frac{1}{k T}\left\langle\left(\frac{\partial E}{\partial \varepsilon^{T}}\right)^{2}\right\rangle+\frac{1}{k T}\left\langle\frac{\partial E}{\partial \varepsilon^{I}}\right\rangle^{2}\right] .
\end{aligned}
$$

In the presence of quadrupolar exchange, the usual strain susceptibility is :

$$
\chi^{\Gamma}=\frac{\partial\left\langle O_{2}^{\Gamma}\right\rangle}{\partial \varepsilon^{\Gamma}}=\frac{B^{\Gamma} \chi_{\mathrm{CEF}}^{\Gamma}}{1-K^{\Gamma} \chi_{\mathrm{CEF}}^{\Gamma}} .
$$

This magnetoelastic coupling gives a contribution to the corresponding elastic constant $C^{I}=C_{0}^{\Gamma}-B^{T} \chi^{\Gamma}$ and :

$$
\left(\frac{C}{C_{0}}\right)^{\Gamma}=\frac{1-G^{\Gamma} \chi_{\mathrm{CEF}}^{\Gamma}}{1-K^{\Gamma} \chi_{\mathrm{CEF}}^{\Gamma}}
$$

vanishes at the quadrupolar ordering temperature $T_{\mathrm{Q}}$ in the case of a second-order transition :

$$
1-G^{\Gamma} \chi_{\mathrm{CEF}}^{\Gamma}\left(T_{\mathrm{Q}}\right)=0
$$

then defines for each CEF scheme the relation between $G^{T}$ and $T_{0}$. In the case of a first order transition, e.g. case of a triplet as ground state [12, 13], $T_{\mathrm{Q}}$ may be defined by the coexistence of both cubic and strained phases. Expression (11) has been extensively used for fitting the softening of sound velocity in Jahn-Teller compounds [14-17].

2.3 PARAstriction. - We propose here a third application of the perturbation theory : parastriction. We limit ourselves to the tetragonal strain mode, that is the dependence of $Q=\left\langle O_{2}^{0}\right\rangle$ on a magnetic field applied along a fourfold axis in the cubic paramagnetic range. Due to symmetry considerations, $\left\langle\mathrm{O}_{2}^{\circ}\right\rangle$ is expanded in even $H$ terms. Second-order calculations lead to :

$\chi^{Q}=\frac{\partial\left\langle O_{2}^{0}\right\rangle}{\partial H^{2}}=\frac{\chi_{\mathrm{CEF}}^{Q}}{\left(1-\left(\theta^{*} / C\right) \chi_{\mathrm{CEF}}^{M}\right)^{2}\left(1-G_{1} \chi_{\mathrm{CEF}}^{T}\right)}$ 
The susceptibility $\chi_{\text {CEF }}^{Q}$ can be calculated from the cubic level scheme without any exchange [18].

$$
\frac{H}{\sqrt{\left|\varepsilon_{3}\right|}}=\frac{1}{\sqrt{\left|\chi^{Q}\right|}} \sqrt{\frac{\left(C_{11}-C_{12}\right)_{0}}{\left|B_{1}\right|}}
$$

exhibits a linear $T$-dependence at high temperature in agreement with the classical high temperature approximation [2]. Its slope gives the magnetoelastic coefficient $B_{1}$. Its extrapolation down to the $T$-axis leads to a paraquadrupolar temperature $\theta_{\mathrm{Q}}$ which strongly depends on $G_{1}$. At low temperature, deviations may exist according to the level scheme. Let us note two possibilities of divergence for second-order transitions : i) $1-\left(\theta^{*} / C\right) \chi_{\mathrm{CEF}}^{M}=0$ at $T=T_{\mathrm{c}}=T_{\mathrm{Q}}$. In this magnetostrictive process the lattice instability is driven by the magnetic dipolar ordering, that is the current case. ii) $1-G_{1} \chi_{\mathrm{CEF}}^{\Gamma}=0$ at $T=T_{\mathrm{Q}}>T_{\mathrm{c}}$. This is the cooperative Jahn-Teller process : the quadrupoles order with a spontaneous strain above the dipolar ordering. That is the same condition as in section 2.2. Note that an analogous treatment may be developped for $B_{2}$ and $G_{2}$ with a threefold field direction.

2.4 ORDERED RANGeS. - In dipolar and/or quadrupolar ordered ranges we diagonalize the whole Hamiltonian (1) in the fourfold axes system with a self-consistent method for the eight average values $\left\langle J_{x}\right\rangle,\left\langle J_{y}\right\rangle,\left\langle J_{z}\right\rangle,\left\langle O_{2}^{0}\right\rangle,\left\langle O_{2}^{2}\right\rangle$ and $\left\langle P_{i j}\right\rangle$ 's : the equilibrium values of the magnetic dipole and quadrupole are then obtained for any direction of application for a magnetic field or an external stress. This method allows us to fully describe both the magnetization and strain mechanisms.

2.5 DETERMINATION OF THE UNKNOWN PARAMETERS. - Among the five parameters $\left(W, x, \theta^{*}, G_{1}, G_{2}\right.$ ) of (1) the cubic CEF ones $(W, x)$ may be known with accuracy from inelastic neutron spectroscopy performed in the paramagnetic range. As discussed in section $2.2, \theta^{*}$ can be derived from $T_{c}$. For the magnetostrictive intermetallics, there then remains two unknown parameters $\left(G_{1}\right.$ and $\left.G_{2}\right)$. Note that only the $G_{1}$ term contributes if the moment points along a fourfold axis and that is the same for the $G_{2}$ term with a threefold moment direction. The main problem is the separation of the two second-order contributions for a same symmetry : $K^{\Gamma}$ and $G^{\Gamma}$ can be obtained from sound velocity data in the cubic range, $B^{\Gamma}$ and $G^{\Gamma}$ both from parastriction and from magnetization and magnetostriction processes in the ordered ranges.

3. Experimental results. - 3.1 COMPETITION BETWEEN THE MAGNETIC DIPOLAR AND QUADRUPOLAR INTERACTIONS. - It is well illustrated by the Jahn-
Teller compound $\mathrm{TmZn}$ [13]. The cubic level scheme is defined by

$$
W=1.2 \pm 0.1 \mathrm{~K} \text { and } x=-0.31 \pm 0.01 .
$$

The ground state is the magnetic triplet $\Gamma_{5}^{(1)}$, the non magnetic doublet $\Gamma_{3}$ lying $37 \mathrm{~K}$ above. The quadrupoles order at $T_{\mathrm{Q}}=8.55 \mathrm{~K}$, the transition to the tetragonal symmetry is of first order. The corresponding $G_{1}$ value is evaluated as $25.5 \mathrm{mK}$, a discontinuity is calculated on the internal energy. The ground state is the $|1\rangle$ level (table I). At $8.12 \mathrm{~K}$, the magnetic dipolar exchange induces a moment on it, the corresponding $\theta^{*}$ being $8.1 \mathrm{~K}$.

Table I. - Matrix elements of the magnetic dipolar and quadrupolar operators for $\Gamma_{5}^{(1)}$ and $\Gamma_{3}$ in cubic symmetry $\left(\mathrm{Tm}^{3+}\right.$ ion $)$.

\begin{tabular}{crcccc} 
& \multicolumn{3}{c}{$\Gamma_{s}^{(1)}$} & \multicolumn{3}{c}{$\Gamma_{3}$} \\
$\left\langle i\left|g_{\mathrm{s}} J_{z}\right| i\right\rangle$ & $|1\rangle$ & $|2\rangle$ & $|3\rangle$ & $|4\rangle$ & $|5\rangle$ \\
$\left\langle i\left|O_{2}^{0}\right| i\right\rangle$ & 0 & +3.15 & -3.15 & 0 & 0 \\
& 15 & -7.5 & -7.5 & +36 & -36
\end{tabular}

This actual $\mathrm{TmZn}$ situation results from a complicated phase diagram : figure 1 gives the dependence of $M=g_{J}\left\langle J_{z}\right\rangle$ and $Q=\left\langle O_{2}^{0}\right\rangle$ on $\theta^{*}$ and $G_{1}$. For weak $G_{1}$ the $\theta^{*}$ term leads to the $|2\rangle$ level as ground state and the $(M \neq 0, Q \sim-5)$ phase is stable. Increasing $G_{1}$ isolates the strongly quadrupolar 1 $1>$ level and the $(M=0, Q \sim 25)$ phase is stabilized. But this phase vanishes in favor of a $(M \neq 0, Q \sim 35)$ one, a moment being induced by the Heisenberg interactions. The higher the $\theta^{*}$ value, the lower the corresponding transition $G_{1}$ value. Note the CEF effects do not lead to $T_{\mathrm{c}}=\theta_{\mathrm{p}}$ for $G_{1}=0$ as explained in section 2.2 and that $T_{\mathrm{c}}$ depends on $G_{1}$ for a given $\theta^{*}$ value. The equality obtained in the actual case of $\mathrm{TmZn}$ is a coincidence.

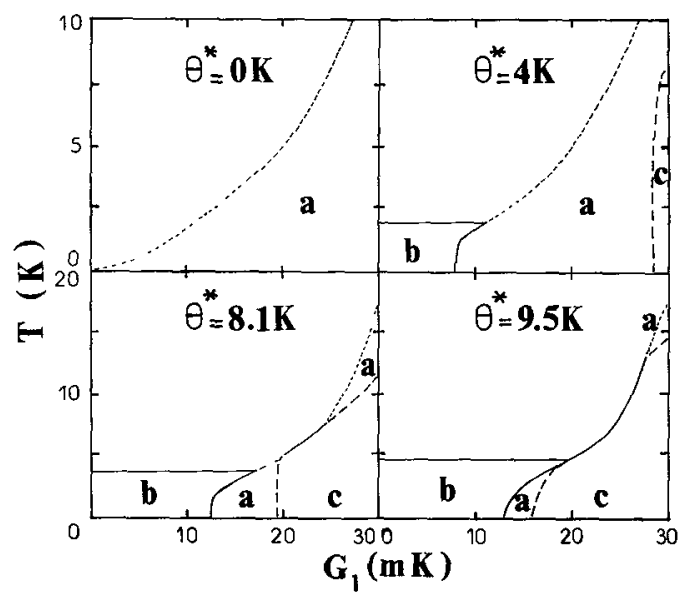

Fig. 1. - Variations of $T_{\mathrm{c}}$ and $T_{\mathrm{Q}}$ as functions of $G_{1}$ for different $\theta^{*}$ in $\operatorname{TmZn}\left(-T_{\mathrm{c}}=T_{\mathrm{Q}} ;--T_{\mathrm{c}} ; \cdots\left(-\ldots T_{\mathrm{Q}}\right)\right.$. Phase a : $(M=0, Q>0) ; \mathrm{b}:(M \neq 0, Q<0) ; \mathrm{c}:(M \neq 0, Q>0)$. 
3.2 Magnetostriction. - 3.2.1 Parastriction. Recent parastriction data have been obtained on TmZn. Applying the magnetic field parallel and perpendicular to the fourfold measurement direction allows elimination of the volume exchange striction. The initial $H^{2}$ behaviour of the $\varepsilon_{3}$ strain has been verified. The temperature dependence of $H / \sqrt{\left|\varepsilon_{3}\right|}$ is given in the figure 2 and compared with the theoretical curve calculated with the $G_{1}$ and $\theta^{*}$ values from section 3.1. The best fit is found with

$$
B_{1}=-27 \pm 2 \mathrm{~K} \text {. }
$$

The difference from the curve calculated without second-order terms $\left(\theta^{*}=8.1 \mathrm{~K}, G_{1}=0\right)$ is obvious : if the high temperature slope is the same, the curvature is opposite in sign at low temperature and the paraquadrupolar temperature $\theta_{\mathrm{Q}}$ is shifted by about $20 \mathrm{~K}$. This proves how fruitful such an experiment can be for determining the unknown quadrupolar exchange coefficient in addition to the magnetoelastic one in magnetostrictive compounds.

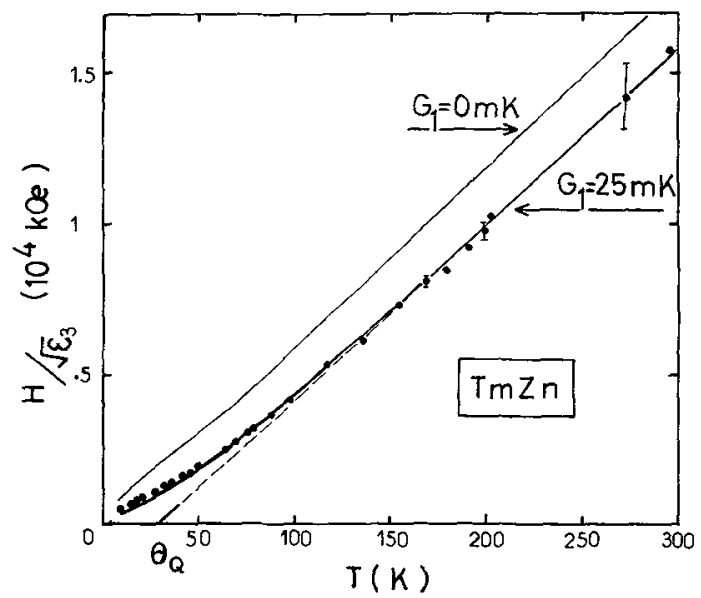

Fig. 2. - Temperature dependence of $H / \sqrt{\left|\varepsilon_{3}\right|}$ in the paramagnetic range of $\mathrm{TmZn}$.

3.2.2 Spontaneous tetragonal strain. - The magnetostrictive compound DyZn [4] orders at $140 \mathrm{~K}$, the magnetization curves show a strong magnetocrystalline anisotropy in favor to the fourfold axis. The tetragonal strain reaches $\varepsilon_{3}=(6.53 \pm 0.12) 10^{-3}$ at $4.2 \mathrm{~K}$ (figure 3). Unfortunately, neutron spectroscopy has not allowed the determination of the spacing of the cubic levels. Extrapolating the CEF parameters variation from the precise Tm- [13], Er-, HoZn [19] values leads to $W=-0.5 \pm 0.2 \mathrm{~K}$ and $x=0.15 \pm 0.1$. Calculations with cubic CEF and bilinear exchange field indicate a threefold easy direction as for any negative $(W, x)$ pair. Thus strong magnetoelastic and quadrupolar effects may help the fourfold axis to become the easy direction. As a result of the large dipolar exchange, $\left\langle O_{2}^{0}\right\rangle$ is found to vary like $M^{2}$ and $B_{1}$ reaches $16 \pm 2 \mathrm{~K} / \mathrm{at}$.

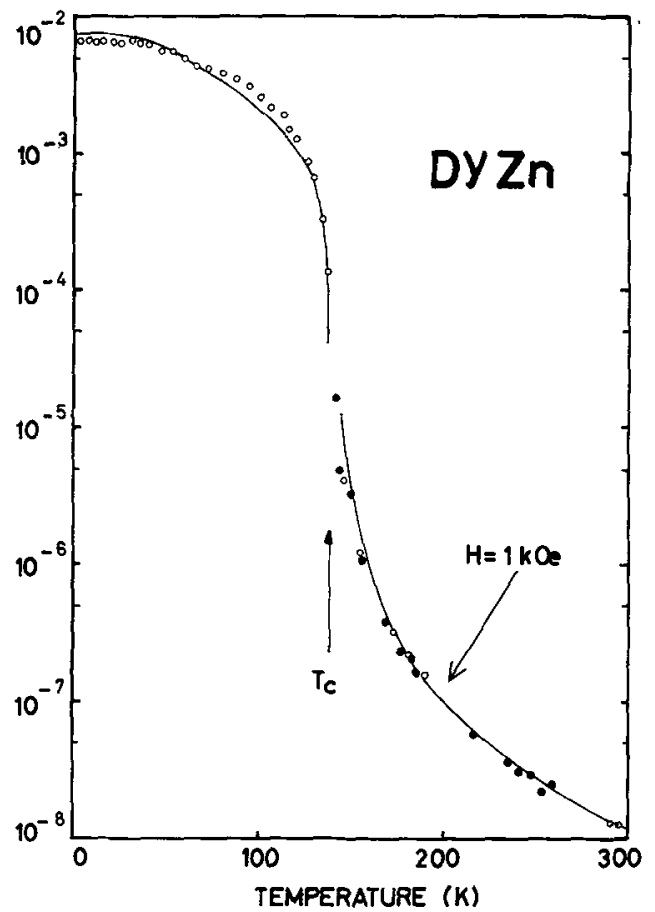

Fig. 3. - Thermal variation of the spontaneous strain $\varepsilon_{3}$ in the ordered state and of the $\varepsilon_{3}$ strain induced by a $1 \mathrm{kOe}$ field in the paramagnetic state in DyZn $(O$, strain-gauge data ; $O$, dilatometric data; full lines are calculated variations).

\subsection{ANALYSIS OF THE MAGNETOELASTIC COEFFICIENT} IN THE RSb AND RZn SERIES. - Such magnetostriction results are available on the $R Z n$ [4] and RSb $[9,20]$ series where the cubic level schemes are known from neutron spectroscopy [21]. We can then calculate the magnetoelastic coefficient $B_{1}$ (tables II and III). From a theoretical point of view, $B_{1}$ is proportional to the strain derivative of the secondorder CEF parameters $\alpha_{\mathrm{J}}\left\langle r^{2}\right\rangle A_{2}, A_{2}$ being the surrounding contribution. If $\partial A_{2} / \partial \varepsilon^{r}$ may be supposed as constant through a series, one can expect $B_{1}$ to vary as $\alpha_{\mathrm{J}}\left\langle r^{2}\right\rangle$.

Table II. - Some magnetoelasticity characteristics in RSb [10, 22].

\begin{tabular}{lllllcc}
\multicolumn{1}{c}{$\mathrm{RSb}$} & $\mathrm{PrSb}$ & $\mathrm{NdSb}$ & $\mathrm{DySb}$ & $\mathrm{HoSb}$ & $\mathrm{ErSb}$ & $\mathrm{TmSb}$ \\
- & - & - & - & - & - & - \\
$T_{\mathrm{N}}(\mathrm{K})$ & - & 16 & 9.5 & 5.5 & 3.8 & - \\
$\varepsilon_{3}\left(10^{-3}\right)(4.2 \mathrm{~K})$ & -0.79 & -2.4 & -5.47 & -2.45 & $0.08(15 \mathrm{kOe})$ & $0.009(15 \mathrm{kOe})$ \\
$B_{1}(\mathrm{~K} / \mathrm{at})$ & -89 & -37 & -35 & -13.8 & +2.5 & +23.5
\end{tabular}


Table III. - Some magnetoelasticity characteristics in RZn.

\begin{tabular}{lcccccc}
\multicolumn{1}{c}{$\mathrm{RZn}$} & $\mathrm{GdZn}$ & $\mathrm{TbZn}$ & $\mathrm{DyZn}$ & $\mathrm{HoZn}$ & $\mathrm{ErZn}$ & $\mathrm{TmZn}$ \\
- & - & - & - & - & - & - \\
$T_{\mathrm{c}}(\mathrm{K})$ & 270 & 200 & 140 & 74 & 20.6 & 8.15 \\
$\varepsilon_{3}\left(10^{-3}\right)(4.2 \mathrm{~K})$ & -0.45 & 4.41 & 6.53 & - & -3.16 & -8.2 \\
$B_{1}(\mathrm{~K} / \mathrm{at})$ & 0 & $15 \pm 1$ & $16 \pm 2$ & $8.4 \pm 0.4$ & $-11.6 \pm 0.6$ & $-25.5 \pm 1$
\end{tabular}

In RSb, this linear dependence is well observed (figure 4). A good agreement is observed also between determinations from sound velocity and strain data, with the exception of PrSb. This confirms the validity of the assumption of neglecting magnetoelastic coefficients of order higher than 2 (section 2.1). In particular the modification of the cubic CEF parameters $A_{4}$ and $A_{6}$ at the ordering point appears to be negligible.

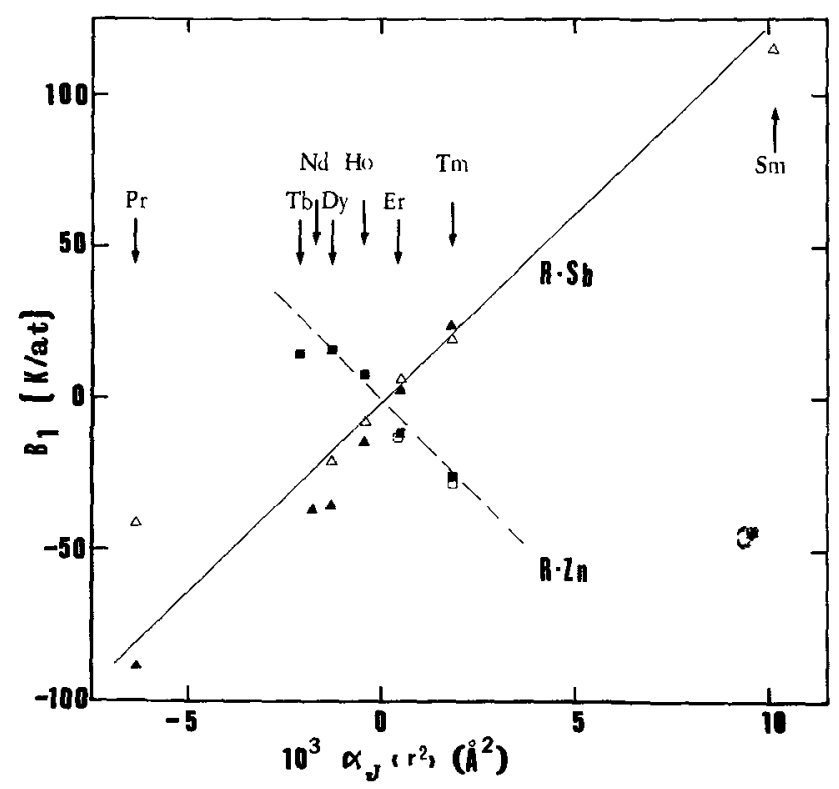

Fig. 4. - Variations of the $B_{1}$ magnetoelastic coefficient in RSb $(\Delta \Delta)$ and $\operatorname{RZn}(\square \square), \Delta \square$ ultrasonic data, $\Delta \cup$ strain data.

In the RZn series the problem is more complicated since a weak tetragonal strain was observed in $\mathrm{GdZn}$ $\left(\varepsilon_{3}=-0.45 \times 10^{-3}\right.$ at $\left.4.2 \mathrm{~K}\right)$ [4]. It has been ascribed to the anisotropic exchange magnetoelastic coupling. Although it modifies negligibly the free energy it may contribute more significantly to the observed strain in other $\mathrm{RZn}$. Being a pure spin effect, it would vary like $\left(g_{\mathrm{J}}-1\right)^{2} J(J+1)$ but we have to note that in a parallel way the $\theta^{*}$ values do not agree with the de Gennes law. For sake of simplicity it was assumed to be constant in reference [4]. The $B_{1}$ variation is then found opposite to the $\alpha_{3}\left\langle r^{2}\right\rangle$ one and the starting assumptions appear to be valid too for the RZn intermetallics.

For analyzing the magnetoelastic coupling, the first attempt is usually to compare the $B_{1} / \alpha_{\mathrm{J}}\left\langle r^{2}\right\rangle$ ratio with the point charge model predictions. Taking into account the eight zinc and six rare earth first neighbours without any shielding factor leads to the following expression of the CEF magnetoelastic coefficient $B_{1}$ for $\mathrm{CsCl}$ type structure [22] :

$$
B_{1}=\frac{e^{2}}{a^{3}}\left(3 q_{\mathrm{RE}}-\frac{64}{9 \sqrt{3}} q_{\mathrm{Zn}}\right) \sqrt{\frac{3}{2}} \alpha_{\mathrm{J}}\left\langle r^{2}\right\rangle .
$$

Assuming $q_{\mathrm{RE}}=3$ and $q_{\mathrm{Zn}}=2$ leads to $B_{1} \simeq+2 \mathrm{~K} / \mathrm{at}$ for the ErZn theoretical value (table III). The same type of discrepancy has been noted for RSb [3]. Since in metallic compounds, the CEF parameters are determined by the electronic structure in the close surroundings of the $4 \mathrm{f}$ ion [19], the difficult theoretical problem of the magnetoelastic coefficients would require first calculating both the direct and exchange coulombic contributions to CEF parameters before studying their strain modifications. A first rough idea is that a tetragonal strain splits the degeneracy of the conduction band ( $e_{\mathrm{g}}$ character) lying close to the Fermi level and thus selects particular wave functions. Another problem is to determine the propagation mechanism which leads to the quadrupolar exchange through the $4 \mathrm{f}$ shell-band coupling.

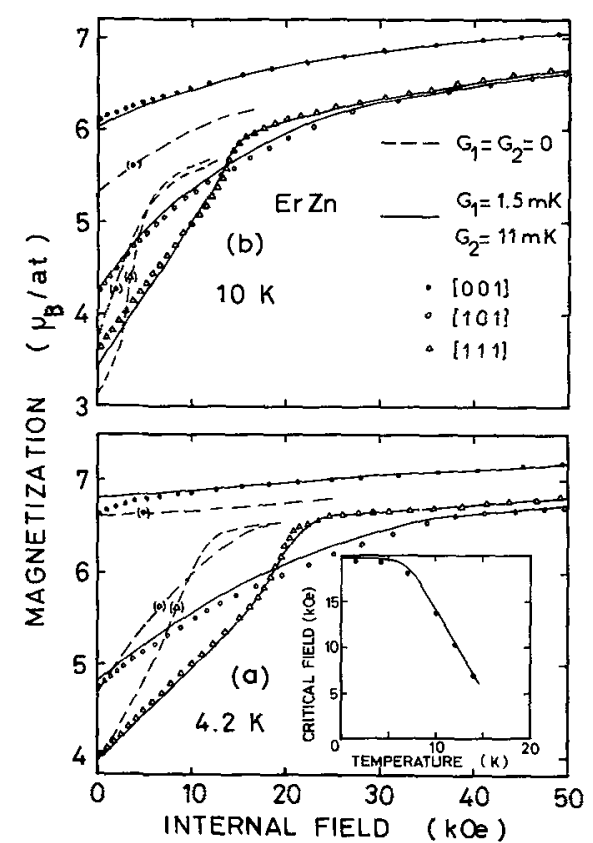

Fig. 5. - Magnetization curves in ErZn along the three principal cubic directions at $(a) 4.2 \mathrm{~K}$ and $(b) 10 \mathrm{~K}$; the inset shows the temperature dependence of the critical field $H_{c}$; the points are experimental data along [101] (O), [111] (A) and [001] (๑); the lines are the theoretical fits (broken curves without second order terms; full curves, with $G_{1}(=1.5 \mathrm{mK})$ and $G_{2}(=11 \mathrm{mK})$ terms $)$. 
3.4 SECOND ORDER CONTRIBUTIONS TO THE MAGNETIZATION PROCESSES. - ErZn orders ferromagnetically at $20.6 \mathrm{~K}$ with a fourfold easy axis [23]. The $\left(\frac{c}{a}-1\right)$ strain reaches $-4.3 \times 10^{-3}$ at $4.2 \mathrm{~K} . W=-0.603 \mathrm{~K}$ and $x=0.16$ are known from neutron spectroscopy. Along [001], the spontaneous moment (only $6.8 \mu_{\mathrm{B}}$ at $4.2 \mathrm{~K}$ instead of $9 \mu_{\mathrm{B}}$ for the free ion value) is strongly purified by field (figure 5). The rotation towards the [101] applied magnetic field is continuous but when the field is applied along [111] the magnetization increases suddenly at a critical value $H_{0}$.

The fit of the magnetization curves without second order terms are shown in figures $5 a$ and $b$ (broken curves) : the magnetization anisotropy is qualitatively described in high magnetic fields; but not the anisotropy of energy. The moment is found to rotate more easily towards the [101] and [111] axes. Including the second order terms $G_{1}=1.5 \mathrm{mK}$ and $G_{2}=11 \mathrm{mK}$ gives a good description of the moment rotation (full curves on the figure 5). It specially explains the sudden increase along the [111] direction at $H_{\mathrm{c}}$ by a stronger variation of the $\left\langle P_{x y}\right\rangle$ average value close to saturation. The $H_{\mathrm{c}}$ temperature dependence is well described (inset).

$$
G_{1}=1.5 \pm 0.5 \mathrm{mK} \text { and } B_{1}=-11.6 \pm 0.6 \mathrm{~K}
$$

obtained from $\varepsilon_{3}$ [3] lead to $K_{1}=0.9 \pm 1.1 \mathrm{mK}$. These values can be compared with determinations from ultrasonic data [24]

$$
\begin{gathered}
\left(\left|B_{1}\right|=12 \pm 1 \mathrm{~K}, \quad K_{1}=2.1 \pm 0.5 \mathrm{mK},\right. \\
\left.G_{1}=2.9 \pm 1.0 \mathrm{mK}\right) .
\end{gathered}
$$

This agreement confirms that the additional terms beside Heisenberg exchange and cubic CEF are mainly those considered here and how necessary they are for fully explaining the magnetization curves.

\section{References}

[1] Cullen, J. R., Blessing, G., Rinaldi, S., Callen, E., J. Magn. Magn. Mat. 7 (1978) 160.

[2] Callen, E., Callen, H. B., Phys. Rev. 139A (1965) 455.

[3] Mullen, M. E., Lüthi, B., WanG, P. S., Bucher, E., LongINotm, L. D., MaIta, J. P., OTt, H. R., Phys. Rev. B 10 (1974) 186

[4] Morin, P., ROUchy, J., DU Tremolet de Lacheisserie, E., Phys. Rev. B 16 (1977) 3182.

[5] Gignoux, D., Grvord, F., Solid State Commun. 21 (1977) 499 and referred papers.

[6] Trammell, G. T., Phys. Rev. 131 (1963) 932.

[7] Stevens, K. W. H., Proc. Phys. Soc. A 65 (1952) 209

[8] LeA, K. R., Leask, M. J. M., Wolf, W. P., J. Phys. Chem. Solids 23 (1962) 1381.

[9] LevY, F., Phys. Kondens. Mater. 10 (1969) 85.

[10] LÜTHI, B., AIP Conf. Proc. 34 (1976) 7. Edts. J. J. Becker and G. H. Lander.

[11] LeVY, P. M., J. Phys. C 6 (1973) 3545.

[12] Kataoka, M., Kanamori, J., J. Phys. Soc. Japan 32 (1972) 113.

[13] Morin, P., Rouchy, J., Schmitt, D., Phys. Rev. B 17 (1978) 3684 .
[14] Kino, Y., Lüthi, B., Mullen, M. E., Solid State Commun. 12 (1973) 275.

[15] Melcher, R. L., Physical Acoustics XII, Edts. W. P. Mason and R. N. Thurston (Academic Press) 1976.

[16] Gehring, G. A., Gehring, K. A., Rep. Prog. Phys. 38 (1975) 1.

[17] Lüthi, B., Mullen, E., Andres, K., Bucher, E., Maita, J. P., Phys. Rev. B 8 (1973) 2639.

[18] Morin, P., SChMtt, D., DU Tremolet de Lacheisserie, E., Phys. Lett. 69A (1978) 217.

[19] For a general view on this problem see

Schmitt, D., J. Phys. F : Metal Physics 7 (1977) 1521 and references therein.

[20] LÜthi, B., WANG, P. S., Wong, Y. H., OtT, H. R., Bucher, E., Second Conf. on C.E.F. effects in metals and alloys (1976) (Plenum Press New York) Edt. A. Furrer.

[21] Bucher, E., Birgeneau, R. J., Maita, J. P., Felcher, G. P., BRUN, T. O., Phys. Rev. Lett. 28 (1972) 746.

[22] Hutchings, M. T., Sol. Stat. Phys. 16 (1964) 277.

[23] Morin, P., Schmirt, D., J. Phys. F 8 (1978) 951.

[24] Morin, P., Waintal, A., Lüthi, B., Proc. 12th Rare Earth Research Conference, Vail (Colorado) 1976. 